Article

\title{
Transcriptome Analysis of Triple Mutant for OsMADS62, OsMADS63, and OsMADS68 Reveals the Downstream Regulatory Mechanism for Pollen Germination in Rice (Oryza sativa)
}

\author{
Eui-Jung Kim ${ }^{1}{ }^{\oplus}$, Woo-Jong Hong ${ }^{1}\left(\mathbb{D}\right.$, Yu-Jin Kim ${ }^{2, *}$ and Ki-Hong Jung ${ }^{1, *}(\mathbb{D}$ \\ 1 Graduate School of Biotechnology \& Crop Biotech Institute, Kyung Hee University, Yongin-si 17104, Korea; \\ alice804@khu.ac.kr (E.-J.K.); hwj0602@khu.ac.kr (W.-J.H.) \\ 2 Department of Life Science and Environmental Biochemistry, and Life and Industry Convergence Research \\ Institute, Pusan National University, Miryang-si 50463, Korea \\ * Correspondence: yjkim2020@pnu.ac.kr (Y.-J.K.); khjung2010@khu.ac.kr (K.-H.J.); \\ Tel.: +82-31-201-3474 (K.-H.J.)
}

Citation: Kim, E.-J.; Hong, W.-J.; Kim, Y.-J.; Jung, K.-H. Transcriptome Analysis of Triple Mutant for OsMADS62, OsMADS63, and OsMADS68 Reveals the Downstream Regulatory Mechanism for Pollen Germination in Rice (Oryza sativa). Int. J. Mol. Sci. 2022, 23, 239. https://doi.org/10.3390/ ijms23010239

Academic Editors: Zsófia Bánfalvi and Yong-Gu Cho

Received: 2 December 2021

Accepted: 25 December 2021

Published: 27 December 2021

Publisher's Note: MDPI stays neutral with regard to jurisdictional claims in published maps and institutional affiliations.

Copyright: () 2021 by the authors. Licensee MDPI, Basel, Switzerland. This article is an open access article distributed under the terms and conditions of the Creative Commons Attribution (CC BY) license (https:/ / creativecommons.org/licenses/by/ $4.0 /)$.

\begin{abstract}
The MADS (MCM1-AGAMOUS-DEFFICIENS-SRF) gene family has a preserved domain called MADS-box that regulates downstream gene expression as a transcriptional factor. Reports have revealed three MADS genes in rice, OsMADS62, OsMADS63, and OsMADS68, which exhibits preferential expression in mature rice pollen grains. To better understand the transcriptional regulation of pollen germination and tube growth in rice, we generated the loss-of-function homozygous mutant of these three OsMADS genes using the CRISPR-Cas9 (clustered regularly interspaced short palindromic repeats-CRISPR associated protein 9) system in wild-type backgrounds. Results showed that the triple knockout $(\mathrm{KO})$ mutant showed a complete sterile phenotype without pollen germination. Next, to determine downstream candidate genes that are transcriptionally regulated by the three OsMADS genes during pollen development, we proceeded with RNA-seq analysis by sampling the mature anther of the mutant and wild-type. Two hundred and seventy-four upregulated and 658 downregulated genes with preferential expressions in the anthers were selected. Furthermore, downregulated genes possessed cell wall modification, clathrin coat assembly, and cellular cell wall organization features. We also selected downregulated genes predicted to be directly regulated by three OsMADS genes through the analyses for promoter sequences. Thus, this study provides a molecular background for understanding pollen germination and tube growth mediated by OsMADS62, OsMADS63, and OsMADS68 with mature pollen preferred expression.
\end{abstract}

Keywords: Oryza sativa; MADS; pollen germination; starch; cell wall; CRISPR-cas

\section{Introduction}

Plants use pollen grains (the male gamete) to deliver sperm cells to the ovary. When the pollen maturing inside the anther reaches the tricellular stage, anthesis and dehiscence occur, and pollen grains are released [1]. To prevent protein damage in this process, the pollen walls fold to maintain a dehydrated state and become temporarily dormant. In addition, when the dehydrated pollen grain blows in the wind and settles in the stigma, it absorbs surrounding water to regain metabolic activity and prepares for pollen germination [2,3]. In the case of rice (Oryza sativa), the viability of pollen disappears if it fails to stick to stigma within five minutes, and since it takes less than ten minutes from germination to tube burst in an in vitro environment, quick and sophisticated mechanisms are required for successful fertilization [4-6]. Furthermore, pollen grains should have enough proteins and energy for successful germination and tube growth. However, the synthesis and accumulation of starch are primarily required to achieve this [7]. Mature pollen contains starch as polysaccharides, sucrose as disaccharides, and glucose as monosaccharides, mainly storing energy sources in the form of starch [7]. Sucrose and starch are 
present inside the dehydrated pollen grain right after anthesis, and energy generated by starch decomposition is used for pollen germination [8]. An appropriate amount of starch must be accumulated in the final stage of pollen maturation, and problems in this process lead to the male-sterile phenotype. The loss-of-function of OsHXK5 encoding hexokinase significantly reduces starch content in pollen and shows male sterility [9]. Furthermore, the mutation of OsSUT1 encoding a sucrose transporter shows a defect in pollen germination and cannot produce any seeds $[10,11]$. In addition, the mature pollen grain must have proteins related to cell wall synthesis and vesicular transport processes, such as exocytosis and endocytosis. The pollen wall is divided into two layers; exine and intine. The exine is composed of sporopollenin, intine is made of cellulose and pectin, but the pollen tube wall is made of a single layer of pectin [12]. Pectin methylesterases (PME) induce pollen wall hardness by demethylesterification of homogalacturonan (HG) pectin component; pectin methylesterases inhibitor (PMEI) inhibit PME and methylesterifies pectin, thereby relaxing the pollen walls to induce elongation [13-15]. Thus, for pollen tube bulging, the cell wall should be relaxed by the interaction between PME and PMEI. Additionally, for rice, 11 OsPMEs and 13 OsPMEI have specifically high expression in the pollen tissue, and exogenic PME treatment induces pollen tube hardness to prevent elongation [16].

MADS-box protein is a transcription factor (TF) with a highly conserved MADS domain and essential functions in floral organ development, seed development, floral organ identity determination, and regulation of flowering time [17-20]. It regulates the expression of downstream genes by directly binding to the CArG motif of the promoter. MADS domain protein is mainly divided into SRF-like MADS domain (Type 1) and MEF2-like MADS domain protein (Type 2). Among the MEF2-like types (also known as MIKC-type), the MIKC ${ }^{*}$-type with a longer intervening domain (I) and duplication of keratin-like domains $(\mathrm{K})$ is well known for its function in pollen development [21]. The MADS-box protein could be dimerized to activate the function as a TF [22]. In the case of Pteridophytes, such as Selaginella moellendorffii and Shorea pallescens, the two groups in S- and P-clades, belonging to MIKC*, mainly form dimers and function as TF [23]. In Arabidopsis thaliana, there have also been studies on AGAMOUS-LIKE (AGL) genes which belong to one of the types of MADS-box. There are five AGL genes (AGL30, AGL65, AGL66, AGL94, and AGL104), which are preferentially expressed in the pollen [24], especially at the tricellular stage after the end of the second mitotic division $[25,26]$. Among them, three combinations of AGL30/66, AGL65/66, and AGL65/104 form dimers and function as TF [26]. MADS directly regulates transcriptional levels by binding to the CArG motif (CC[A/T]6GG) in the promoters of the downstream genes $[27,28]$. Although MADS binds to the serum response element (SRE)-type CArG box, CC[A/T]6GG, it can be attached to MEF2-CArG box, C[A/T]8G or CTA[A/T]4. For example, in Arabidopsis thaliana, AGL30/66, AGL65/66, and AGL65/104 dimerization forms have a higher preference for the MEF2-CArG box. In addition, genes specifically expressed in pollen at the tricellular stage are better preserved in MEF2-CArG boxes than in the SRE-CArG boxes based on their $3 \mathrm{~kb}$ upstream promoter [29].

In the case of Oryza sativa, OsMADS62, OsMADS63, and OsMADS68 have specifically high expression in the pollen grains at the tricellular stage; OsMADS62 and OsMADS63 belong to the MIKC* S-clade, whereas OsMADS68 belongs to the MIKC* P-clade [30]. These three proteins can form homodimers and heterodimers through diverse combinations, and the interactions of OsMADS62/68 and OsMADS63/68 are the strongest [30]. Pollen viability and germination defects were identified in RNAi mutants with reduced OsMADS62 or OsMADS68 expression in the osmads63 mutant background [30]. However, the defects were observed at the heterozygote state with a mixture of normal and defective pollens. Thus, detailed phenotypic observation and related regulatory mechanisms have not been sufficiently investigated. Here, we generated homozygous triple loss-of-function mutants of three OsMADS (box) genes belonging to the MIKC*-type (OsMADS62, OsMADS63, and OsMADS68) that are preferentially expressed in mature pollen grains. By analyzing the total RNA of mature anther through RNA-seq analysis, we identified differentially expressed genes (DEG) in the triple loss-of-function mutant anther versus wild-type anther. Furthermore, 
we conducted functional classification analyses using gene ontology (GO) enrichment, MapMan, and Kyoto Encyclopedia of Genes and Genomes tools. Down(-regulated) DEGs are closely associated with biological processes for pollen tube development, such as starch accumulation and cell wall synthesis. Because OsMADS62, OsMADS63, and OsMADS68 function in the late stage of pollen maturation, germination, and tube elongation, transcriptome analysis using triple mutations should provide an important molecular basis for future research.

\section{Results}

\subsection{OsMADStri Pollen Cannot Germinate, Resulting in a Male-Sterile Phenotype}

The recent transcriptome analysis identified three MADS-box genes in rice, OsMADS62, OsMADS63, and OsMADS68, which had a high expression in mature anther and pollen grains [30]. In the CAFRI-rice database, the three abovementioned genes showed similar expression patterns: weak expression in panicle, intermediate expression in mature anther, and strong expression in mature pollen grains (Figure 1A). However, these three OsMADS genes showed low expression in the anthers at the late tetrad, young microspore, and vacuolated microspore stages (Figure 1B). However, the expression increased a little in mature anther, including tricellular pollen, and was the strongest in pollen grain, right after anther dehiscence. Among three OsMADS genes, OsMADS68 had the highest expression and OsMADS62 had the lowest expression. Furthermore, OsMADS62 and OsMADS63 shared higher sequence similarities compared with OsMADS68. It has been reported that the three genes have functional redundancy on late pollen development. For overcoming the functional redundancy and the functional analysis of these genes, we generated the lossof-function homozygous triple mutants by targeting three genes simultaneously using the CRISPR-Cas9 (clustered regularly interspaced short palindromic repeats-CRISPR associated protein 9) system. The first exons of OsMADS63 and OsMADS68 and the fifth exon of OsMADS62 were targeted for gene editing. One base (thymine) was inserted in the fifth exon of OsMADS62 and the first exon of OsMADS68, and one base (adenine) was also inserted in the first exon of OsMADS63; this loss-of-function mutation was named OsMADStri (Figure 1C). All mutations resulted in an early stop codon, causing an abnormal protein. The T0 generation of the OsMADStri had a sterile phenotype without seeds. Unlike the wild-type (WT) Dongjin plants that produce seeds over $90 \%$, the T0 generation of OsMADStri cannot produce seeds and showed a complete sterile phenotype (Figure 1D,E,R). The reciprocal crossing assay with control WT plants showed that OsMADStri has a defect in developing male reproductive tissues. When OsMADStri is used as the pollen donor, the control plants cannot produce any seeds, but it can produce heterozygous progeny when used as the pollen recipient (Figure S1). OsMADStri did not show any difference in anther size compared with the WT (Figure 1F,G). However, in the case of iodine potassium iodide solution $\left(I_{2} K I\right)$ staining, the OsMADStri pollen grains were not stained at the tricellular stage (Figure 1H,I). Likewise, the difference could be observed in Ruthenium red and Calcofluor white staining (Figure 1J-M). This indicates that acidic polysaccharides constituting pectin and cellulose of the intine layers of OsMADStri were abnormal. The size and shape of OsMADStri mature pollen grains seemed identical to those of the WT. However, the OsMADStri pollen showed abnormal germination patterns and could not elongate any pollen tube under in vitro germination (Figure $1 \mathrm{~N}-\mathrm{Q}, \mathrm{S}$ ). As the defects were identified at the late pollen development for male-female organ interaction, we expect these three MADS-box TFs to play key regulatory roles in the developmental process. 


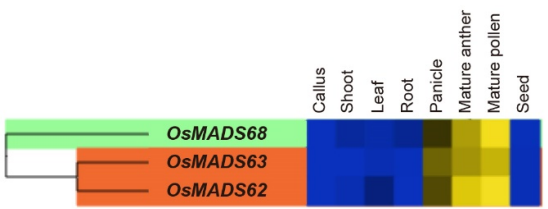

C
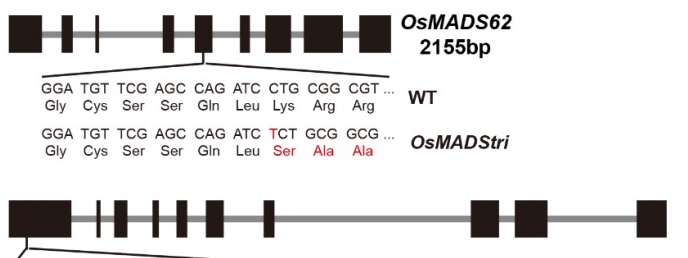

GTG ACG TTC TCC AAG CGG CGA AAC GGG ... WT
Val Thr Phe Ser Lys Arg Arg Asn Gly

GTG ACG TTC TCC AAG CGG CGA AAA CGG

OSMADStri

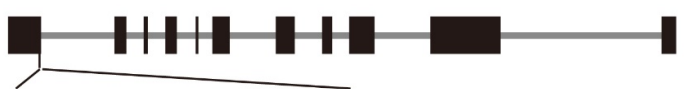

AAC GAC AAG CCC ACG ATT TGC GTT GGC ... WT
Asn Asp Lys Pro Thr lle Cys Val Gly

AAC GAC AAG CCC ACG ATT TGT CGT TGG
Asn Asp Lys Pro Thr lle Cys Arg Trp
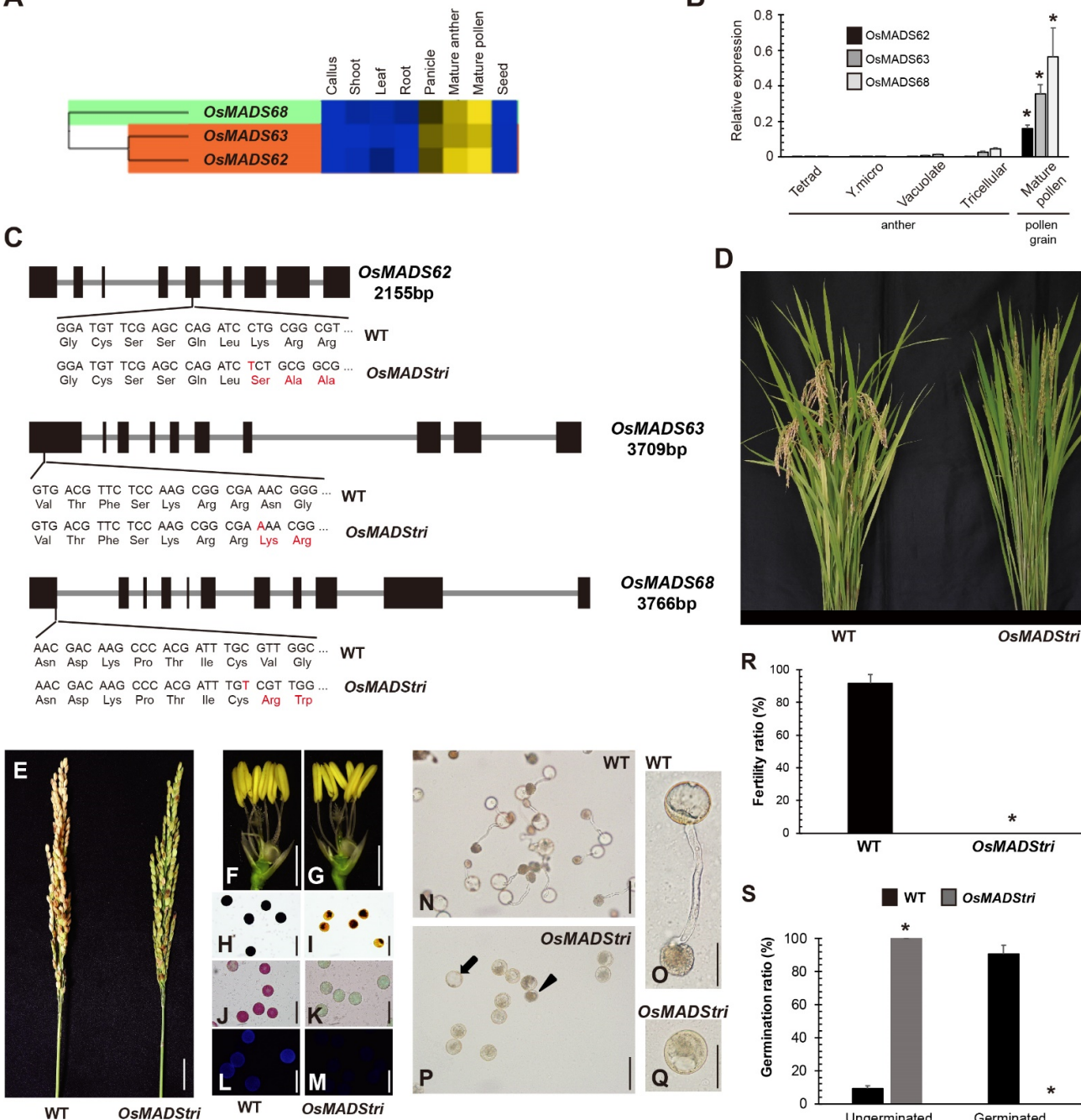

OsMADS68 3766bp

Figure 1. The mutation target site and phenotype of OsMADStri. (A) Gene expression pattern of three OsMADS using CAFRI-rice. Leaf, 7 days before flowering to 7 days after flowering; Shoot and Root, 7 days after germination; Panicle, 7 days before flowering; Mature anther, anther at anthesis period. Heatmap displays the expression value according to the color gradation. Blue is the lowest, and yellow is the highest expression value. (B) The transcriptome expression pattern of the three OsMADS genes in anther at various developmental stages and pollen grains after anther dehiscence. Tetrad, the anther that contains mitotic pollens at late tetrad stage; Y. micro, the anther that contains pollens at young microspore stage; Vacuolate, the anther that contains pollens at vacuolated microspore stages; Tricellular, mature anther with trinucleate pollen; Mature pollen, the pollen grains right after anther dehiscence. Error bars indicate the standard deviation and $p$-value calculated by one-way Analysis of Variance (ANOVA). ${ }^{*}, p<0.01$. (C) OsMADS62, OsMADS63, and OsMADS68, the target genes of OsMADStri. The gene size is written below its name by base pairs. The fifth exon of OsMADS62 was a target site, and thymine was inserted. The first exon of OsMADS63 and OsMADS68 were mutated by one base insertion, respectively. bp, base-pair; WT, wild-type. (D) The phenotype of the whole plant. Bars $=10 \mathrm{~cm}$. (E) The panicle pictures of WT and OsMADStri. Bars $=2 \mathrm{~cm}$. $(\mathbf{F}, \mathrm{G})$ The pictures of reproductive tissue of WT and OsMADStri. Bars $=2 \mathrm{~mm}$. (H-M) Histochemical staining of the mature pollen grain of WT and OsMADStri. $(\mathbf{H}, \mathbf{I})$ is the iodine potassium iodide staining, $(\mathbf{J}, \mathbf{K})$ is Ruthenium red staining, $(\mathbf{L}, \mathbf{M})$ is Calcofluor white staining. Bars $=80 \mu \mathrm{m}$. (N-Q) In vitro germination is performed in solid pollen germination media. Black arrow and triangle indicate hydrated and unhydrated pollen grains, respectively. Bars $=80 \mu \mathrm{m}, 40 \mu \mathrm{m}$. (R,S) Fertility ratio and germination ratio of WT and OsMADStri. Error bars indicate the standard deviation and $p$-value calculated by one-way Analysis of Variance (ANOVA). *,$p<0.01$. 


\subsection{Transcriptome Analysis Suggests the Potential Downstream Genes of OsMADS62, OsMADS63, and OsMADS68}

We tried to identify downstream genes of OsMADS62, OsMADS63, and OsMADS68 using WT and OsMADStri line anthers. RNA-seq analysis was performed with the anthers sampled at the tricellular stage of WT and OsMADStri. Differentially expressed genes (DEG) were selected based on three criteria; (1) genes with absolute $\log _{2}$ value (OsMADStri anther/WT anther; fold change/FC) was $\geq 1$, (2) genes which $p$-value of $\log _{2}$ (FC) was $\leq 0.05$ in three biological replicates, and (3) the mean expression value in WT tricellular anther sample was $\geq 10$. Out of the total 55,986 rice genes, there were 8159 DEGs: 4734 upregulated genes and 3425 downregulated genes (Figure 2A). Additionally, DEGs were refined by selecting (1) genes having preferential expression in mature anthers and pollen grains compared with eight other organs, callus, leaf, root, seed, shoot, pre-flowering panicle, anther, and pollen grains, through K-means clustering analysis and (2) genes for which absolute value of $\log _{2}(F C)$ was $\geq 2$. DEGs were selected based on their anatomical expression patterns because the three OsMADS genes had a highly specific expression in mature anther and pollen grains. The DEGs selected were expressed not only in wildtype anther but also in wild-type pollen grain. As a result, 274 genes were selected as anther (preferred) upregulated genes, and 658 genes were selected as anther (preferred) downregulated genes (Figure 2A).

\subsection{Downregulated Genes in OsMADStri Anther/Pollen Mainly Show GO Enrichment Related to Cell Wall Biosynthesis and Vesicle Secretion Processes}

GO enrichment analysis was conducted to identify biological processes closely associated with these mature anther DEGs, and results are as follows: amino acid transport (GO:0006865), translation (GO:0006412), and metabolic process (GO:0008152) were the most enriched in anther upregulated genes. However, cell wall modification (GO:0042545), clathrin coat assembly (GO:0048268), and phosphatidylinositol metabolic process (GO:0046488) were the most enriched in anther downregulated genes (Figure 2B,C). Interestingly, GOs, such as clathrin coat assembly (GO:0048268), cation transport (GO:0006730), and microtubule-based movement (GO:0007018), were only enriched in anther downregulated genes (Figure 2C). They are mainly related to endocytosis/exocytosis, which is essential for pollen tube elongation.

\subsection{MapMan Analysis for Downregulated Genes in OsMADStri Further Supports the Significance of Genes Related to Cell Wall Synthesis}

MapMan analysis also supported the results of GO analysis (Figure 3 and Figure S2). Using the MapMan toolkit, we analyzed four overviews associated with mature anther DEGs: regulation, metabolism, cellular response overview, and biotic and abiotic stress overview. Of the 274 anther upregulated genes, 40 were mapped to the regulation overview. Furthermore, 18 signaling receptors, 8 TF, 8 genes related to protein degradation, 3 genes related to post-translational modification, and 3 genes related to hormone metabolism were identified (Figure 3A). The signaling receptors included domain unknown functions and glycoprotein; TF included AP2/EREBP, bHLH, MYB, and WRKY; protein degradationrelated genes included a serine protease, ubiquitin, and E3 ligase. Among 658 anther downregulated genes, 137 were mapped to the regulation overview, and there were 25 signaling receptors, $23 \mathrm{TF}, 12$ calcium signaling-related genes, and $6 \mathrm{G}$-protein signaling-related genes. Signaling receptors included leucine-rich repeat and Catharanthus roseus-like RLK1 (CrRLK1L), TF included AP2/EREBP, C2H2 zinc finger, MADS, MYB, and NAC, and calcium signing-related genes included calcium-dependent protein kinase (CPK) and calmodulinlike (CML). Additionally, anther upregulated genes did not have a significantly enriched feature in the metabolism overview (Figure 3B). However, 45 genes of the anther downregulated genes were closely related to cell wall modification and organization, including 11 PMEs and 8 pectate lyases. In addition, anther downregulated genes included four peroxidase precursors, two glutaredoxins, and two thioredoxins (Figure 3C,D), indicating 
that the ROS signaling process might play an important role in the downstream pathways of OsMADS62, OsMADS63, and OsMADS68.

A

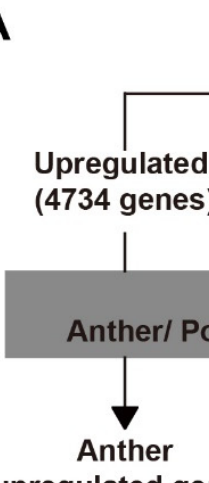

upregulated genes

(274 genes)

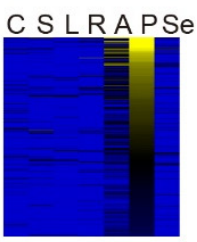

$|\log 2(\mathrm{FC})|>2.0$,

RNA-seq

-specific expression pattern

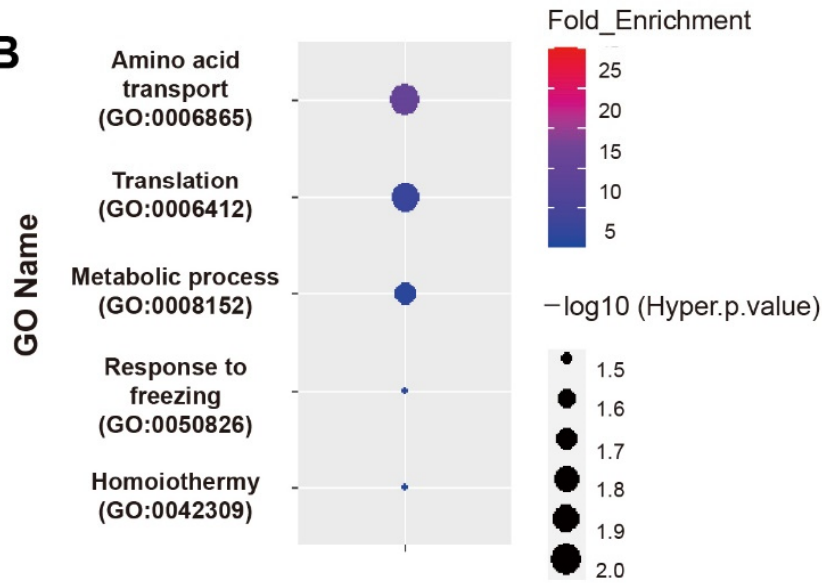

Anther upregulated genes
C

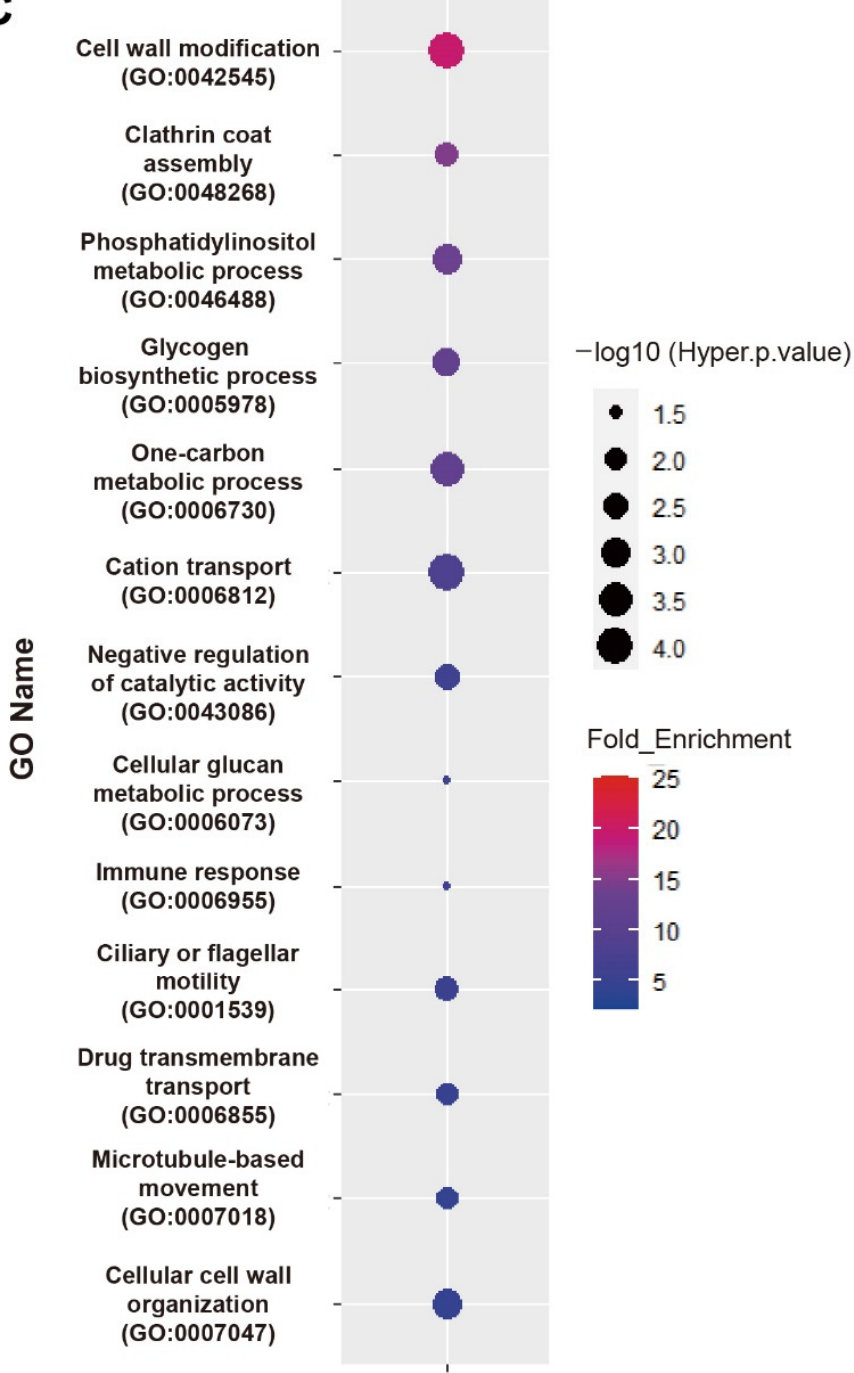

Anther downregulated genes

Figure 2. Schematic of DEG screening and GO annotation graph. (A) This schematic diagram shows how we screened Differentially Expressed Genes in mature pollen grains (pollen DEGs) after conducting RNA-seq. Four thousand, seven hundred and thirty-four upregulated genes and 3425 downregulated genes were selected, and the expression patterns of each tissue were compared, and the absolute value of $\log _{2}(F C)$ was 2.0 or higher was selected in detail. Thus, 274 genes were finally selected as pollen upregulated genes and 658 genes as pollen downregulated genes. The heatmap was drawn based on the RNA-seq value of each tissue in wild-type plants, yellow indicates a high expression, and blue indicates a low expression. C, Callus; S, Shoot; L, Leaf; R, Root; A, Anther; P, Pollen grains, Se, Seed. (B,C) GO enrichment annotation of pollen DEGs. The color represents the fold enrichment of GO, and the size of the circle represents the hyper $p$-value. The GO number was written under GO Names (terms), sorted in descending order based on the fold enrichment.

\subsection{Literature Searches of the Downregulated Genes in OsMADStri Suggest Their Potential} Function for Future Studies

Among the 658 anther downregulated genes, 324 were in funricegenes, of which 52 are registered with MapMan (Table 1). Cell wall-related genes were the most enriched, with 11 OsPMEs (OsPME10, OsPME12, OsPME14, OsPME16, OsPME23, OsPME26, OsPME27, OsPME32, OsPME33, OsPME35, OsPME41), and cellulose synthase-like D 3 (CSLD3). 
Regarding calcium regulation, five OsCPKs (OsCPK14, OsCPK21, OsCPK25, OsCPK26, OsCPK27), four CMLs (CML2, CML3, CML28, CML32), and Calcium-dependent protein kinase 2 (CDPK2) were included. Additionally, SWEET5, which is involved in sugar and ion transport, and genes encoding G-protein coupled complex, such as OsRopGEF2, OsRopGEF8, and OsRopGEF3, also belonged to the anther downregulated genes. The function of OsRopGEF in late pollen development has been previously reported [31]. The double loss-of-function mutant of OsRopGEF2 and OsRopGEF8 showed a phenotype with reduced fertility [31].

A

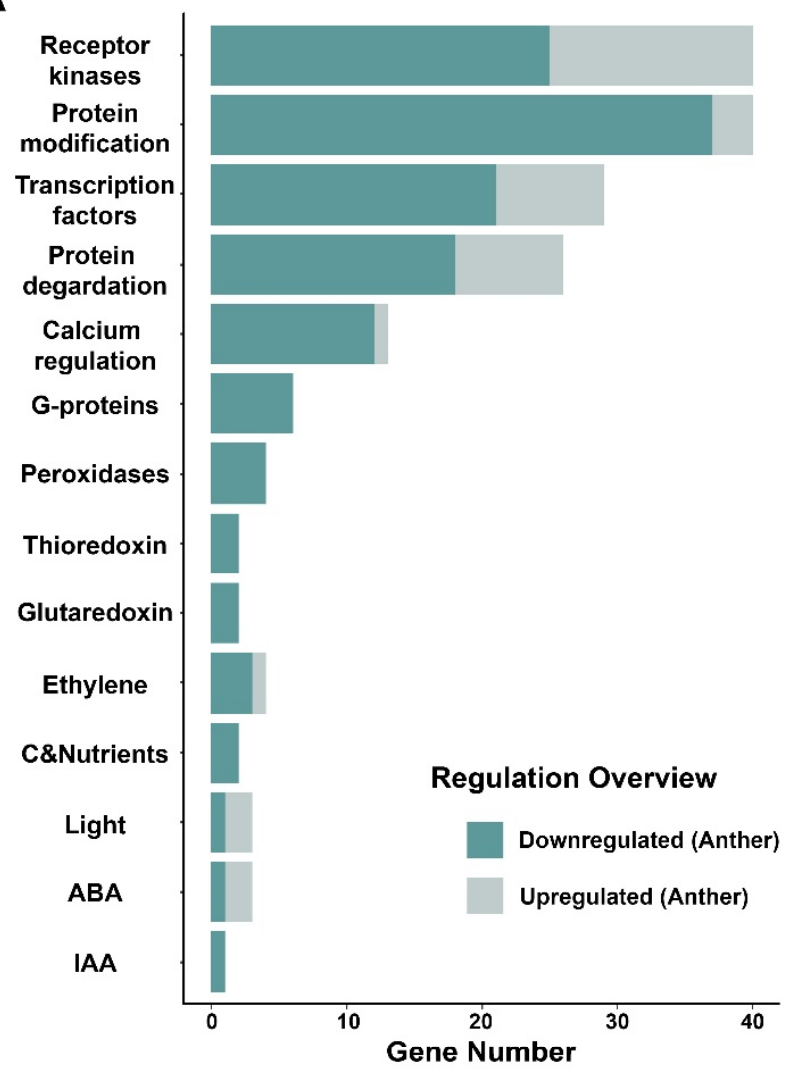

B

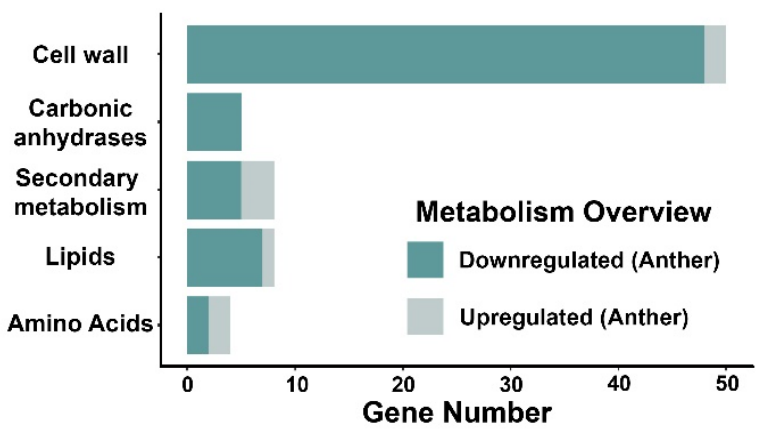

C

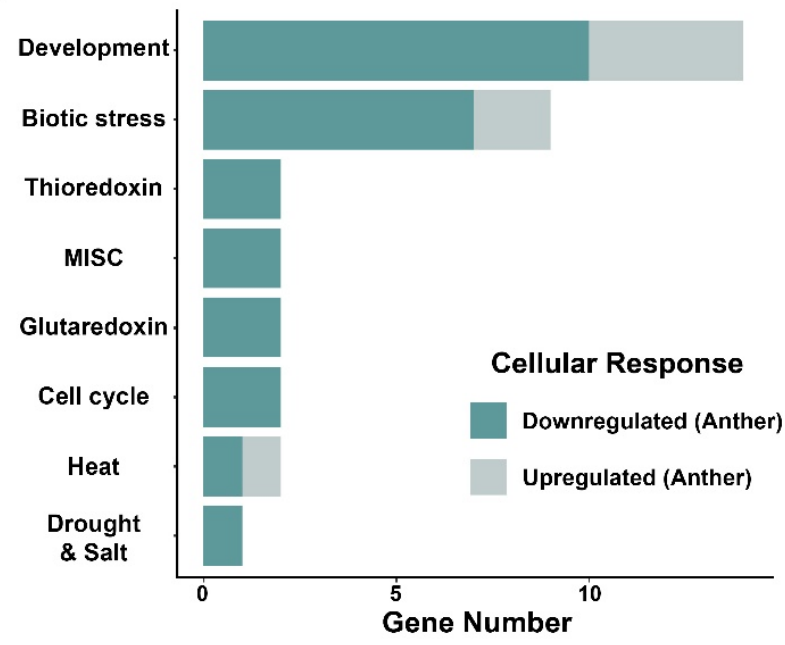

D

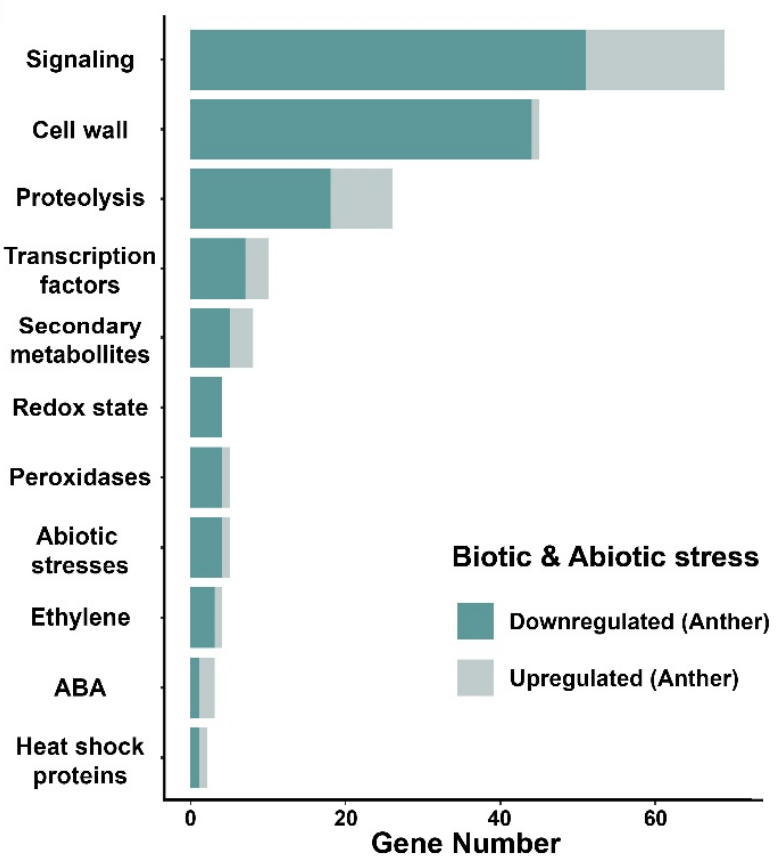

Figure 3. MapMan analysis of DEGs in anther. Each graph is the degree of enrichment for the MapMan pathway. (A) is the regulation overview, (B) is the metabolism overview, $(\mathbf{C})$ is the cellular response, and (D) is the biotic and abiotic stress among the MapMan pathway. The $\mathrm{x}$-axis represents the gene number, and the $y$-axis represents the molecular function registered in MapMan. Dark green indicates the anther downregulated genes, and light green is the anther upregulated genes. 
Table 1. The GO annotation and MapMan analysis of known genes in anther downregulated genes of OsMADStri.

\begin{tabular}{|c|c|c|c|c|c|}
\hline DEG & Locus & Gene Name & MapMan & GO Number & GO Name \\
\hline Down & LOC_Os12g43700 & PR1-121 & \multirow[b]{2}{*}{ Biotic stress } & Non & Non \\
\hline Down & LOC_Os08g23460 & OsSTA209 & & Non & Non \\
\hline Down & LOC_Os12g30150 & OsCPK27 & \multirow{11}{*}{$\begin{array}{l}\text { Calcium } \\
\text { regulation }\end{array}$} & GO:0007186 & $\begin{array}{l}\text { G-protein coupled receptor } \\
\text { protein signaling pathway }\end{array}$ \\
\hline Down & LOC_Os10g27170 & OsSTA242 & & Non & Non \\
\hline Down & LOC_Os12g03970 & OsCPK26 & & GO:0006468 & $\begin{array}{l}\text { protein amino acid } \\
\text { phosphorylation }\end{array}$ \\
\hline Down & LOC_Os11g04170 & OsCPK25 & & GO:0006468 & $\begin{array}{l}\text { protein amino acid } \\
\text { phosphorylation }\end{array}$ \\
\hline Down & LOC_Os12g12730 & CML28 & & GO:0001539 & ciliary or flagellar motility \\
\hline Down & LOC_Os08g04890 & CML32 & & GO:0001539 & ciliary or flagellar motility \\
\hline Down & LOC_Os08g42750 & OsCPK21 & & GO:0006468 & $\begin{array}{l}\text { protein amino acid } \\
\text { phosphorylation }\end{array}$ \\
\hline Down & LOC_Os05g41270 & OsCPK14 & & GO:0006468 & $\begin{array}{l}\text { protein amino acid } \\
\text { phosphorylation }\end{array}$ \\
\hline Down & LOC_Os01g59360 & CDPK2 & & GO:0006468 & $\begin{array}{l}\text { protein amino acid } \\
\text { phosphorylation }\end{array}$ \\
\hline Down & LOC_Os11g03980 & CML2 & & GO:0001539 & ciliary or flagellar motility \\
\hline Down & LOC_Os12g03816 & CML3 & & GO:0001539 & ciliary or flagellar motility \\
\hline Down & LOC_Os11g45720 & OsPME33 & \multirow{24}{*}{ Cell wall } & GO:0042545 & cell wall modification \\
\hline Down & LOC_Os12g37660 & OsPME35 & & GO:0042545 & cell wall modification \\
\hline Down & LOC_Os12g36040 & EXPA26 & & GO:0007047 & cellular cell wall organization \\
\hline Down & LOC_Os04g54850 & OsPME16 & & GO:0042545 & cell wall modification \\
\hline Down & LOC_Os11g43830 & OsPME32 & & GO:0042545 & cell wall modification \\
\hline Down & LOC_Os04g39590 & FLA14 & & Non & Non \\
\hline Down & LOC_Os07g49100 & OsPME23 & & GO:0042545 & cell wall modification \\
\hline Down & LOC_Os08g25710 & CSLD3 & & GO:0030244 & cellulose biosynthetic process \\
\hline Down & LOC_Os09g26360 & OsPME27 & & GO:0042545 & cell wall modification \\
\hline Down & LOC_Os04g38560 & OsPME14 & & GO:0042545 & cell wall modification \\
\hline Down & LOC_Os03g28090 & OsPME12 & & GO:0042545 & cell wall modification \\
\hline Down & LOC_Os03g18860 & OsPME10 & & GO:0042545 & cell wall modification \\
\hline Down & LOC_Os08g34910 & OsPME26 & & GO:0042545 & cell wall modification \\
\hline Down & LOC_Os02g03550 & XTH26 & & GO:0005975 & carbohydrate metabolic process \\
\hline Down & LOC_Os09g37360 & OsPME41 & & GO:0042545 & cell wall modification \\
\hline Down & LOC_Os08g13980 & OsSTA207 & & GO:0005975 & carbohydrate metabolic process \\
\hline Down & LOC_Os02g26320 & FLA20 & & Non & Non \\
\hline Down & LOC_Os09g22090 & OsSTA225 & & GO:0005975 & carbohydrate metabolic process \\
\hline Down & LOC_Os06g08810 & OsSTA172 & & GO:0045226 & $\begin{array}{l}\text { extracellular polysaccharide } \\
\text { biosynthetic process }\end{array}$ \\
\hline Down & LOC_Os11g08400 & OsSTA253 & & Non & Non \\
\hline Down & LOC_Os10g35930 & OsPLIM2c & & Non & Non \\
\hline Down & LOC_Os03g27610 & OspPLAIIbeta & & GO:0006629 & lipid metabolic process \\
\hline Down & LOC_Os12g07874 & OsSTA276 & & GO:0006412 & translation \\
\hline Down & LOC_Os05g51090 & SWEET5 & & GO:0006813 & potassium ion transport \\
\hline Down & LOC_Os01g55520 & OsRopGEF8 & \multirow{3}{*}{ G-proteins } & Non & Non \\
\hline Down & LOC_Os05g48640 & OsRopGEF2 & & Non & Non \\
\hline Down & LOC_Os02g17240 & OsRopGEF3 & & Non & Non \\
\hline Down & LOC_Os06g45240 & OsSTA177 & $\begin{array}{l}\text { Receptor } \\
\text { kinases }\end{array}$ & GO:0006468 & $\begin{array}{l}\text { protein amino acid } \\
\text { phosphorylation }\end{array}$ \\
\hline Down & LOC_Os05g38980 & OsNOX4 & Redox state & GO:0055114 & oxidation reduction \\
\hline
\end{tabular}


Table 1. Cont.

\begin{tabular}{cccccc}
\hline DEG & Locus & Gene Name & MapMan & GO Number & GO Name \\
\hline Down & LOC_Os01g40400 & HDA701 & & GO:0016575 & $\begin{array}{c}\text { histone deacetylation } \\
\text { multicellular organismal } \\
\text { development }\end{array}$ \\
Down & LOC_Os11g32960 & OsSTA261 & & GO:0007275 & Non \\
Down & LOC_Os10g32900 & OsCCT34 & & Non & regulation of transcription \\
Down & LOC_Os04g38770 & MADS61 & Transcription & GO:0045449 & Non \\
Down & LOC_Os04g46670 & OsSTA129 & factors & Gon & regulation of transcription, \\
Down & LOC_Os01g11350 & OsbZIP02 & & GO:0006355 & DNA-dependent \\
Down & LOC_Os11g43740 & MADS68 & & GO:0045449 & regulation of transcription \\
rown & LOC_Os08g38590 & MADS62 & & GO:0045449 & regulation of transcription \\
Down & LOC_Os12g22630 & OsSTA279 & & GO:0045449 & Non \\
Down & LOC_Os07g31830 & OsSTA196 & & & Non transcription \\
\hline
\end{tabular}

\subsection{Genes Expected to Be Directly Regulated by OsMADS among Anther Downregulated Genes}

We identified genes that are directly regulated by three OsMADS among the anther downregulated genes. Since the MADS TF specifically binds to the CArG motif, genes having at least one CArG motif in the promoter of anther downregulated genes were selected. One hundred and fifty-six genes had a CArG motif in the $2 \mathrm{~kb}$ upstream promoters since their expression was decreased by OsMADStri (Figure 4A). The CArG motif consisted of ten base pairs, and DEGs having CTATAAATAG in the promoter were the most; however, DEGs with CTAATATTAG were the least (Figure 4B). When MapMan overview analysis was performed on the 156 genes, 15 were related to cell wall modification and organization, 11 to protein regulation, 9 to receptor kinases, 8 to TF (Figure $4 \mathrm{C}$ and Table 2). Finally, this result suggests that OsMADS62, OsMADS63, and OsMADS68 can directly regulate candidate genes related to protein regulation, transcriptional regulation, kinase mediating signaling, and cell wall modification and organization pathways.

A

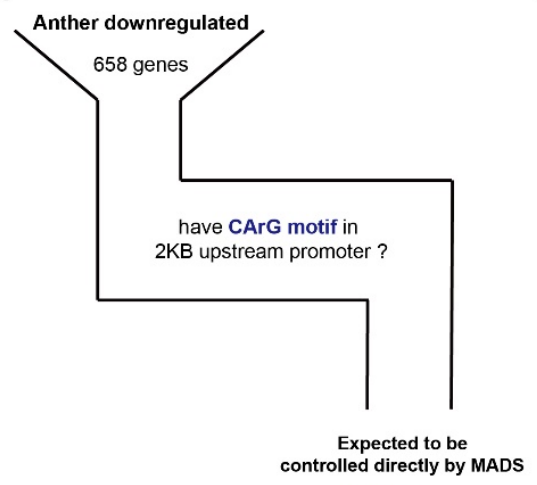

B

\begin{tabular}{cc}
\hline $\begin{array}{c}\text { CArG motif } \\
\text { (+ strand) }\end{array}$ & Gene number \\
\hline CTA TAAA TAG & 23 \\
CTA TTTT TAG & 22 \\
CTA AAAA TAG & 21 \\
CTA TATA TAG & 14 \\
CTA AATT TAG & 14 \\
CTA AAAT TAG & 12 \\
CTA AATA TAG & 12 \\
CTA ATTT TAG & 12 \\
CTA TTTA TAG & 11 \\
CTA ATTA TAG & 11 \\
CTA TATT TAG & 7 \\
CTA ATAA TAG & 6 \\
CTA TAAT TAG & 6 \\
CTA TTAA TAG & 5 \\
CTA ATAT TAG & 3 \\
\hline Total & 179 \\
\hline
\end{tabular}

C

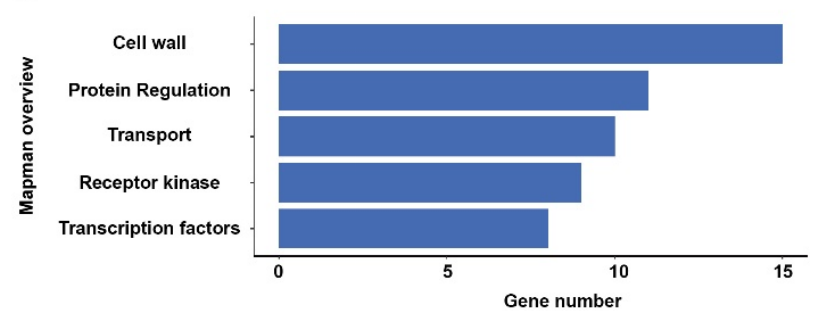

156 genes

Figure 4. Screening and analyzing the CArG Cis-regulatory elements motif in the promoter of anther downregulated genes. (A) Of the 658 anther downregulated genes, 156 genes had CArG motifs in $2 \mathrm{~kb}$ (2000 base-pair) upstream of the promoter regions. The upstream were numbered based on the adenine of the start codon. (B) Types and frequencies of CArG motifs existed in the promoter of anther downregulated genes. (C) MapMan enrichment analysis for 156 DEGs that had CArG motif in the promoter region. The $x$-axis means gene number, and the $y$-axis represents the type of overview registered in MapMan. 
Table 2. The promoter analysis of anther downregulated genes in OsMADStri mutant.

\begin{tabular}{|c|c|c|c|c|c|}
\hline Locus ID & Gene Name & GO & Functions & $\log 2 \mathrm{FC}$ & Sequence \\
\hline LOC_Os11g45720 & OsPME33 & cell wall modification & Cell wall & -19.25 & CTAAAATTAG \\
\hline LOC_Os01g55440 & CIPK30 & signal transduction & Protein modification & -17.43 & CTAATTTTAG \\
\hline LOC_Os05g29740 & OsPMEI23 & homoiothermy & PME & -16.10 & CTAAAATTAG \\
\hline LOC_Os05g11790 & CIPK20 & signal transduction & Protein modification & -15.79 & CTAAAAATAG \\
\hline LOC_Os08g07600 & OsPROPEP2 & Non & Signaling & -13.86 & CTATAATTAG \\
\hline LOC_Os11g08400 & OsSTA253 & Non & Development & -13.50 & CTATAAATAG \\
\hline LOC_Os10g35930 & OsPLIM2c & Non & Development & -13.16 & CTATATTTAG \\
\hline LOC_Os01g20970 & OsPMEI3 & Non & PME & -11.68 & CTAAAATTAG \\
\hline LOC_Os11g32960 & OsSTA261 & $\begin{array}{c}\text { multicellular organismal } \\
\text { development }\end{array}$ & Transcription factor & -11.54 & CTAAAAATAG \\
\hline LOC_Os02g02450 & YSL7 & transport & Transport & -11.50 & CTAAAAATAG \\
\hline LOC_Os02g58660 & OsSTA85 & cation transport & Transport & -10.99 & CTATATATAG \\
\hline LOC_Os10g32900 & OsCCT34 & Non & Transcription factor & -10.00 & CTATAATTAG \\
\hline LOC_Os08g23130 & OsOPT5 & Non & Transport & -9.76 & CTAAAAATAG \\
\hline LOC_Os12g12860 & OsCPK29 & glycogen biosynthetic process & Protein modification & -9.51 & CTAAATTTAG \\
\hline LOC Os01g13710 & YSL1 & pathogenesis & Transport & -9.31 & CTAAAAATAG \\
\hline LOC_Os02g33840 & OsSTA64 & Non & Protein modification & -9.17 & CTAAAAATAG \\
\hline LOC_Os02g09450 & OsGDPD11 & lipid metabolic process & Lipid & -9.07 & CTAAAATTAG \\
\hline LOC_Os05g38980 & OsRBOH4 & oxidation reduction & Redox & -8.87 & CTAAATATAG \\
\hline LOC_Os02g33740 & OsSTA63 & Non & Protein modification & -8.47 & CTAATTATAG \\
\hline LOC_Os03g10550 & OsSTA94 & Non & Non & -8.24 & CTATTTATAG \\
\hline LOC_Os06g06430 & OsSTA169 & Non & Non & -7.46 & CTAAATATAG \\
\hline LOC_Os02g31950 & OsSTA59 & Non & Non & -5.46 & CTAAAAATAG \\
\hline LOC_Os02g51730 & OsSTA78 & Non & Redox & -5.46 & CTAATAATAG \\
\hline LOC Os02g02460 & YSL8 & transport & Transport & -4.85 & CTAAATTTAG \\
\hline LOC_Os03g16840 & OsSTA95 & Non & Non & -3.68 & CTAATATTAG \\
\hline LOC_Os05g20150 & OsMTD4 & ino acid phosp & Signaling & -3.51 & CTAATTTTAG \\
\hline LOC Os02g03550 & XTH26 & carbohydrate metabolic process & Cell wall & -3.30 & CTAATTTTAG \\
\hline LOC_Os02g01990 & OsCCT02 & Non & Non & -3.06 & CTATTTATAG \\
\hline LOC_Os05g28530 & OsGRL10 & cell redox homeostasis & Redox & -3.00 & CTATATTTAG \\
\hline LOC_Os08g13980 & OsSTA207 & carbohydrate metabolic process & Cell wall & -2.86 & CTAAAAATAG \\
\hline LOC_Os12g07700 & ISC14 & iron-sulfur cluster assembly & Protein modification & -2.78 & CTAAATATAG \\
\hline LOC_Os06g08810 & OsSTA172 & carbohydrate metabolic process & Cell wall & -2.16 & CTATATTTAG \\
\hline LOC_Os07g31830 & OsSTA196 & Non & Transcription factor & -2.13 & CTATTAATAG \\
\hline LOC_Os01g03640 & OsLPR5 & oxidation reduction & Redox & -2.02 & CTATTTATAG \\
\hline
\end{tabular}

\subsection{Starch Metabolism-Related Genes Whose Expression Is Significantly Reduced in OsMADStri}

A mature pollen grain of OsMADStri was not stained with $I_{2} \mathrm{KI}$ dye. Since $I_{2} \mathrm{KI}$ stains starch among polysaccharides in plants, we assume that the mature pollen grain of OsMADStri might have defects in the starch accumulation. Therefore, we tried to find genes related to starch function among downregulated genes in OsMADStri. First, using the funricegene database, which provides information on rice genes with known functions [32], 132 genes were selected, including starch synthase, 14-3-3 protein, hexokinase, pyruvate kinase, and sucrose transporter. Four genes (LOC_Os02g01590, LOC_Os03g01750, LOC_Os05g51090,LOC_Os06g40120) were selected for further analyses because they have a significant expression level in WT mature anther but are extremely downregulated in OsMADStri. The largest was the $\log _{2} \mathrm{FC}$ (OsMADStri anther/WT anther, fold change) for LOC_Os05g51090, a SWEET gene well known as a sugar transporter. The $\log _{2} \mathrm{FC}$ value was -3.0. The $\log _{2} \mathrm{FC}$ in the other three genes, LOC_Os02g01590 encoding OsVIN2, LOC_Os03g01750 encoding polysaccharide binding phosphatase, and LOC_Os06g40120 encoding SPX-domain-containing protein, were about -2.0. A similar tendency of reduction was confirmed in expression verification using real-time quantitative reverse transcription-polymerase chain reaction (RT-qPCR) (Figure 5). The expression value of LOC_Os02g01590 was 0.27 at WT but decreased nine times to 0.03 at OsMADStri. Moreover, the expression value of LOC_Os03g01750 decreased 49.4 times, and the expression value of LOC_Os06g40120 decreased 7.87 times, and the expression value of LOC_Os05g51090 was 4.06 at WT but decreased about 12 times to 0.33 at OsMADStri. 

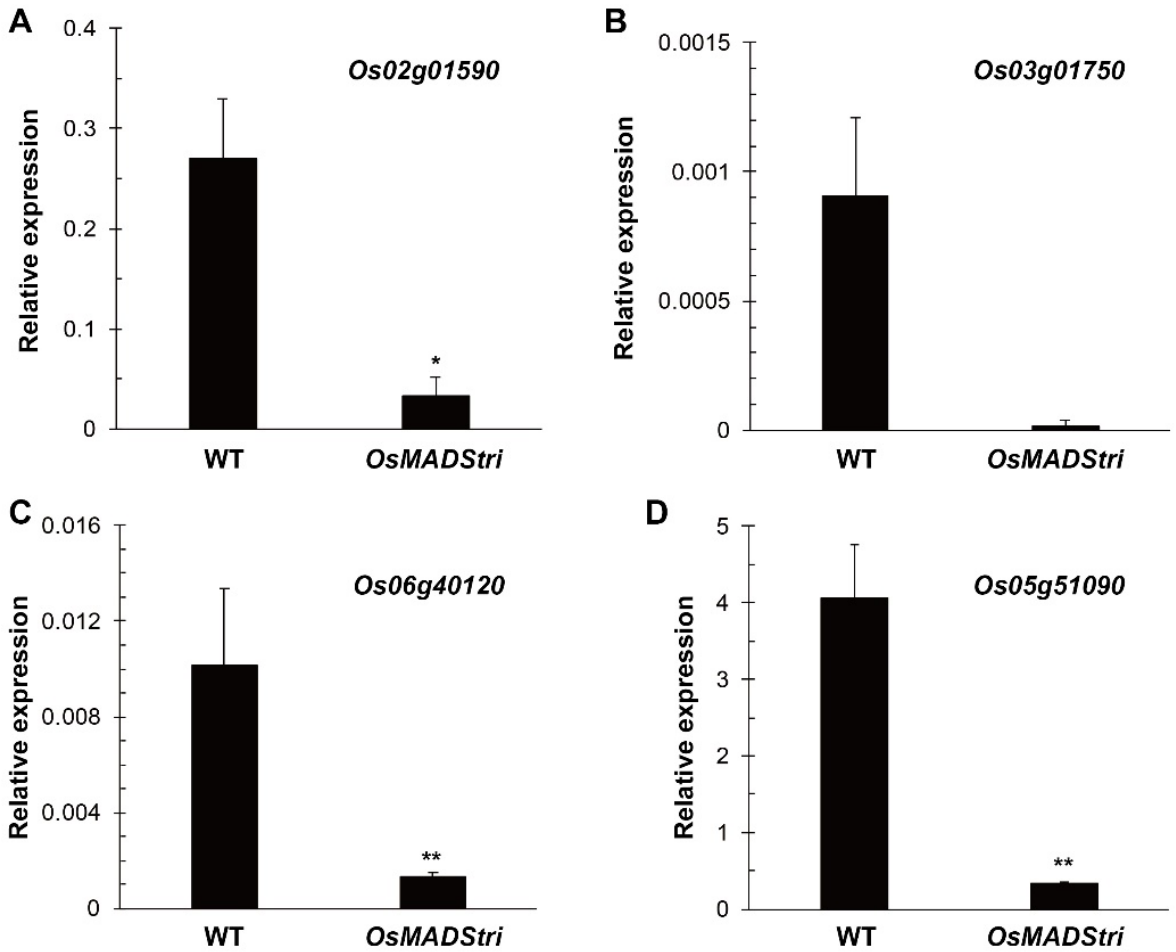

Figure 5. Verifying the expression patterns of four genes (A) LOC_Os02g01590, (B) LOC_Os03g01750, (C) LOC_Os06g40120 and (D) LOC_Os05g51090 related to starch accumulation among downregulated genes. The expression value of those genes was verified by RT-qPCR. OsUBI5 (LOC_Os01g22490) was used as an internal control. Error bars indicate the standard deviation of three biological replicates. $p$-value calculated by one-way Analysis of Variance (ANOVA) with repeated measurements using Tukey's pairwise comparison test. ${ }^{*}, p<0.1{ }^{* *}, p<0.01$.

\section{Discussion}

Based on a previous study, we showed that functional redundancy among OsMADS62, OsMADS63, and OsMADS68 might exist because OsMADS68RNAi, OsMADS62RNAiosmads63, and OsMADS68RNAiosmads63 did not show a complete pollen defect [30]. Additionally, all observations and assays in the study were conducted in heterozygotes, so the molecular and genetic investigation was highly restricted [30]. In addition, studies on genes that function in the pollen maturation process have many limitations. The mutant that has a defect in pollen maturation and tube germination processes cannot participate in fertilization, so it is impossible to obtain homozygous mutants except by using gene-editing techniques [33,34]. Using the CRISPR-Cas9 system, we simultaneously knocked out three OsMADS (OsMADS62, OsMADS63, OsMADS68) highly expressed in mature pollen grains. Then, we generated the homozygous mutants for all three genes, OsMADStri; furthermore, OsMADStri shows a complete male-sterilizing phenotype. This result indicates that multiple gene-editing systems can easily overcome functional redundancy among three pollen-preferred OsMADS genes. Next, we analyzed the transcriptome data using the mature anther samples of OsMADStri, compared to the corresponding wild-type anther. It is ideal to collect only pollen grains from mutant and wild type for transcriptome analysis, but our transcriptome analysis had technical limitations because the number of OsMADStri mutant is not sufficient to collect the suitable number of samples for the transcriptome analysis. Therefore, we tried to enrich the target tissue by collecting the mutant anthers. Thereafter, the technical problem was supplemented through transcriptomic data of wildtype pollen grains and mature anthers which we previously produced [35]. By applying this transcriptome data to the OsMADStri DEGs isolated in this study, we confirmed that most of these DEGs were also preferentially expressed in pollen grains (Figure 2A) Furthermore, by analyzing the global transcriptome of the mature anther of OsMADStri, we identified 
many potential downstream genes or biological processes regulated by OsMADS. Among them, cell wall modification and clathrin coat assembly were the most enriched in anther downregulated genes. This suggests that OsMADS62, OsMADS63, and OsMADS68 might regulate pollen tube growth through cell wall modification and intercellular trafficking of molecules or enzymes during tube growth.

\subsection{OsMADStri Is Defective in the Late Maturation Stage of Pollen}

Under $I_{2} K I$ staining, pollen grains in the OsMADStri were partially stained, and starch did not accumulate sufficiently in the pollen grains. Furthermore, OsMADStri showed abnormal intine and pectin staining, and it could not germinate. This was significantly different with ruptured phenotypes from other male-sterile mutant lines [16,33,34]. Most male-sterile mutants have a defect in tapetum by unusual programmed-cell death (PCD), which affects early pollen development consisting of young microspore, vacuolated pollen, and binucleate pollen, particularly pollen outer wall development, and causes problems in the entire pollen grains of the mutants [36-38]. Likewise, mutant lines that have problems before the first mitosis stage show a shrunken and burst pollen grain phenotype due to abnormal pollen wall formation [36-38]. Starch filling occurs in the late bicellular microspore stage after the first mitosis [8]. Moreover, the expression of these OsMADS was very low in the early pollen development consisting of a tetrad (stage 8), young microspores (stage 9), and vacuolated microspores (stage 10) stages, and the expression patterns were confirmed by qRT-PCR. Based on these expression patterns, we expect that three OsMADS genes play important roles in the pollen maturation process or the later processes. Because OsMADStri pollen cannot induce a germination and tube elongation step, it seems that OsMADStri already had a defect at the pollen maturation stages. Rather, the phenotype of OsMADStri was similar to that of the Rice Immature Pollen 1 (RIP1) mutant (rip1). RIP1 functions in the pollen maturation stage, and its mutations had male-sterile phenotype and abnormalities in the starch accumulation and intine layers [39]. There was no difference in WT and rip1 in the pollen grain size, the internal vacuole did not disappear, and the cytoplasmic density remained low in rip1 [39]. However, since there was no significant difference in the expression of RIP1 in OsMADStri, RIP1 functions upstream of the three $M A D S$ s, or in the different pathway with that of the three $M A D S s$, further study is required.

\subsection{The Low Expression of OsVIN2 and SPX Genes in OsMADStri Would Prevent Starch Accumulation}

Expression of three OsMADS with specifically high gene expression in pollen grains were observed in pollen but not in anther walls [30]. It had very low expression at stage 10, and as they reach stage 11-12, the expression gradually increased [30]. Therefore, it can be assumed that these three MADS TFs are associated with the development of the late stages of pollen maturation. Furthermore, OsMADStri was defective in starch accumulation and was well correlated with the developmental expression feature. In the case of control plants, starch highly accumulated in pollen grains at the mature (tricellular) stage [8]. Furthermore, our study confirmed the result in the homozygous mature pollen of OsMADStri. To explain the molecular base, we listed downregulated genes in OsMADStri mature pollen grains and discovered that four genes were related to the starch metabolism pathway. Unexpectedly, they did not have specifically high expression in pollen grains. The four genes might be mainly involved in sugar transport and flux with SWEET, OsVIN2, polysaccharide binding phosphatase, and SPX-domain connecting protein. OsVIN2 is a well-known gene that regulates the size and weight of seeds due to the flux regulation of sucrose $[9,40]$. In addition, OsSPX1 regulates the expression of genes encoding hexokinase and sugar transporter [41]. LOC_Os06g40120-encoding SPX-domain that connects protein should have similar functions in mature pollen with OsSPX1. Starch is stored in the late stage of pollen maturity, and for this process, sugar should be provided through the anther wall and tapetum [42,43]. However, our RNA-seq analysis revealed that OsMADStri had the reduced expression of genes related to sugar transport and sucrose flux. Therefore, it might 
cause insufficient starch accumulation during the pollen maturation, resulting in the male sterility of OsMADStri.

3.3. PME Is Related to Cell Wall Organization and It Controls the Concentration of Calcium Ions Inside Pollen Grains

OsMADStri pollen grains exhibited abnormal staining in Ruthenium red and Calcofluor white. Ruthenium red stains acid polysaccharides, a constituent of pectin, making it possible to check whether the pectin layer of pollen grains is normal [44]. Calcofluor white stains cellulose and is used to identify the intine layer of pollen grains [33,45]. In addition, since the intine layer of the pollen wall is composed of cellulose and pectin, we can say that of OsMADStri is abnormal [46]. The size of OsMADStri pollen grain was similar to that of WT pollen; it was the form of an intact sphere, and the anther dehiscence occurred normally. Additionally, we tried to determine the molecular reason for this abnormality in the OsMADStri mutant through RNA-seq analysis. The genes related to the cell wall synthase pathway, vesicle secretion, and calcium signaling were significantly reduced in OsMADStri. PME was the most obvious gene family associated with anther downregulated genes in OsMADStri. 11 OsPMEs (OsPME10, OsPME12, OsPME14, OsPME16, OsPME23, OsPME26, OsPME27, OsPME32, OsPME33, OsPME35, OsPME41) were downregulated in OsMADStri. PME demethylesterifies pectin, hardens cell walls, and mediates the interactions between calcium ions and pectin [16,47]. Demethylesterified HG by PME allows ionic bonds to be formed between calcium ions and carbonyl groups of HG, through which external calcium ions can pass the intine to activate calcium signaling [14,47,48]. After hydration, calcium ions can be accumulated in the pollen grain, especially where the tube emerges, and it remains at a high concentration until the germination begins [49]. Calcium ions cannot pass through the pollen wall because of the low expression of the 11 OsPME genes in OsMADStri, and it might cause an abnormality in calcium signaling. However, even if the calcium ion penetrates the pollen grain, it might be difficult for calcium signal transduction to occur. This is because the expression of kinases, such as OsCPK, $C M L$, $C D P K$, also decreased in OsMADStri. Additionally, OsCPK21 is known to be involved in the late-stage development of pollen [41]. The expression of the three OsMADS analyzed in this study was decreased in the loss-of-function mutant of OsCPK21, and OsCPK21 in OsMADStri was also decreased by nine times. This indicates that OsCPK21 and three OsMADS genes may indirectly mutually affect the transcriptional regulation [41].

\subsection{A Model for Downstream Genes Transcriptionally Affected by the OsMADS62, OsMADS63, and OsMADS68 Genes}

First, when downregulated genes in OsMADStri were analyzed, they could be grouped into three main groups. The first was DEGs involved in starch metabolism, including OsVIN2, SWEET, and SPX-domain containing protein gene. Second, DEGs involved in cell wall synthesis include EXPA, CSLD, XTH, and 11 OsPMEs. Finally, PME, CDK, CDPK, $C M L$, and $C I P K$ are involved in calcium ion-meditating signaling pathways and pectin crosslink in the pollen grain. More interestingly, the Ruptured Pollen tube (RUPO), belonging to CrRLK1L, a plant-specific acceptor-like kinase, was identified as a downregulated gene with $-6.78 \log _{2} \mathrm{FC}$ in OsMADStri. Additionally, RUPO is located in the cell membrane and vessel of the apical region of the pollen tube and plays a role as a regulator of potassium carriers [50]. Altogether, a transcriptional regulation model mediated by OsMADS62, OsMADS63, OsMADS68 is suggested in Figure 6. However, our transcriptome work has a limitation: exactly how DEGs are affected by each of OsMADS62, OsMADS63, and OsMADS68 or combination among them is unclear. Furthermore, since OsMADS TF forms a heterodimer for the function, it might be required to analyze the interaction between the promoter of key DEGs and the combination of three OsMADS proteins through further studies. Unlike dicotyledonous plants, such as Arabidopsis thaliana, rice has one ovule in each flower. Since it is impossible for mutant pollen grain to compete with the wildtype pollen grain and complete the fertilization process, there was a limitation in that heterozygous lines available through current biotechnology or breeding techniques had to 
be used. As a result, few studies have been published. With the CRISPR/Cas9 system, it is possible to produce homozygous mutants which enable to carrying out detailed genetic and molecular analyses on pollen maturation or pollen tube germination in rice. The study of transcriptional analysis using the OsMADStri in this study is expected to be a very useful resource for understanding the overall regulatory process for the maturation and germination of rice pollen mediated by OsMADS62, OsMADS63, and OsMADS68.

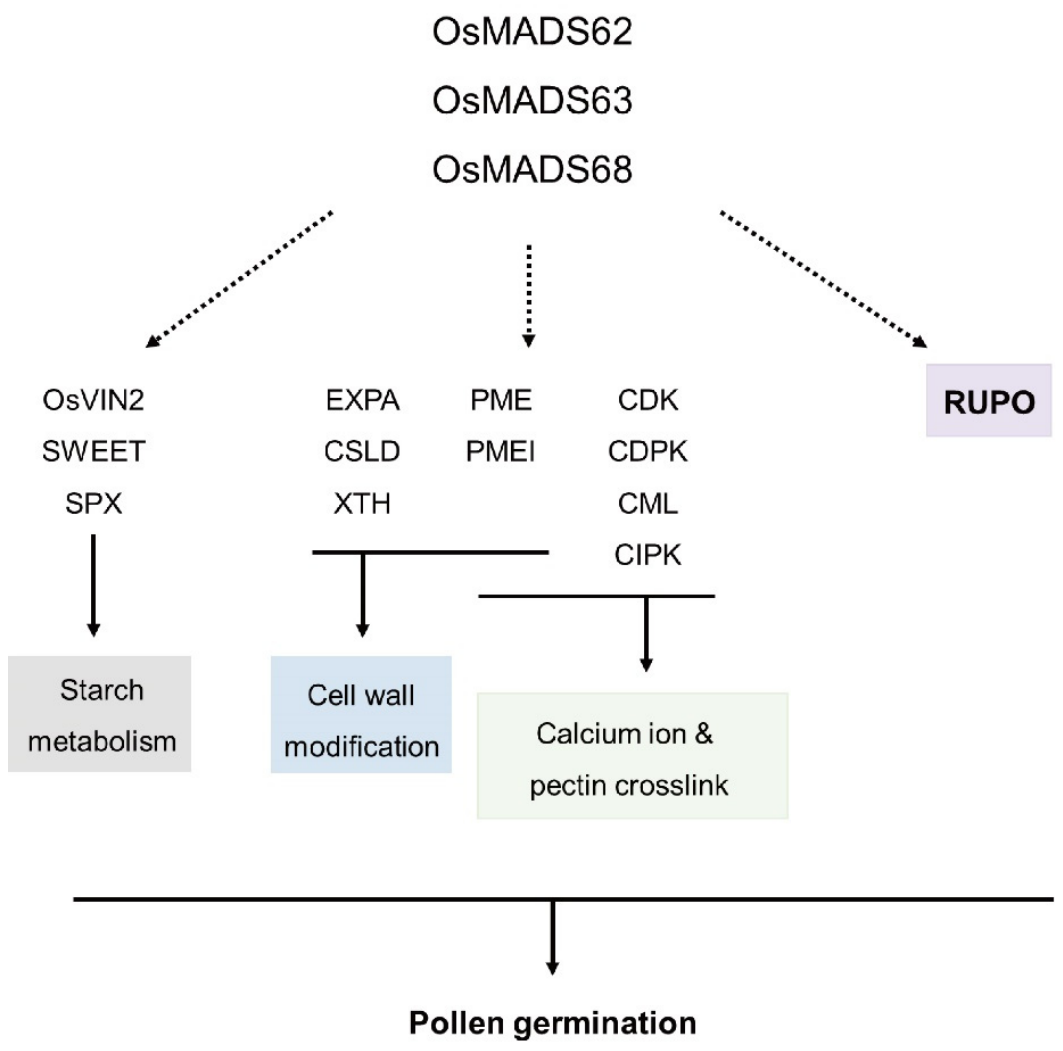

Figure 6. The model of downstream genes in three OsMADS.

\section{Materials and Methods}

\subsection{Plant Materials and Growth Conditions}

WT rice (O. sativa japonica cv. Dongiin) seeds were sterilized with $50 \%$ of sodium hypochlorite and washed with distilled water and germinated on Murashige and Skoog media ( $\mathrm{pH}$ 5.7) under a growth chamber in 7 days $\left(28 / 25^{\circ} \mathrm{C}\right.$ day/night) [51]. The seedlings were grown in the greenhouse for three weeks and transferred to a paddy field at the Kyung Hee University. In the case of OsMADStri, since the mutant showed a completely sterile phenotype, the transgenic T0 plant was ratooned and maintained for two years in the greenhouse and paddy field. Transplanting was conducted at the end of May 2020 and 2021, and seeds were harvested at the end of October.

\subsection{Nucleic Acids Extraction and RT-qPCR}

For genotyping analysis, flag leaves of transgenic plants were sampled, frozen in liquid nitrogen, and ground using a Tissue-Lyser II (Qiagen, Hilden, Germany). DNA was extracted using the CTAB (cetyltrimethylammonium bromide)-chloroform method [52]. In addition, sequencing analysis for genotyping was performed by Macrogen (https: / / dna.macrogen.com, accessed on 4 January 2021), using the BigDye Terminator v3.1 cycle sequencing kit (Applied Biosystems). For total RNA extraction, WT and mutant anther tissues were sampled by freezing in liquid nitrogen. After grinding the frozen sample with liquid nitrogen using a pestle and mortar (CoorsTek 60310), total RNA was extracted using TRIzol buffer and 1-bromo-3-chloropropane (BCP) and purified using 
the RNeasy plant mini kit (Qiagen, Hilden, Germany). Finally, the complementary DNA (cDNA) was synthesized using SuPrime Script RT premix (GeNet Bio). To identify the tissue-specific expression by quantitative real-time PCR (RT-qPCR), we used the Roter-Gene $\mathrm{Q}$ instrument system (Qiagen, Hilden, Germany) and the internal control for rice ubiquitin 5 (OsUbi5, LOC_Os01g22490), as previously reported [53]. RT-qPCR was performed with three independent biological replicates. A previously reported method was used to calculate the relative transcript levels and fold change [54]. All RT-qPCR primers used in our experiments are listed in Table S1.

\subsection{Vector Construction and Plant Transformation}

First, to design the guide RNA for CRISPR-Cas9 vector cloning, we selected two target regions using the CRISPRdirect software, and the synthesized oligo dimer was ligated to the pRGEB32 binary vector [55]. Next, the modifying plasmid vector was amplified in Escherichia coli; TOP10, and then transformed into the Agrobacterium tumefaciens, LBA4404. Induced rice callus was co-cultivated with Agrobacterium cells and incubated on a 2N6$\mathrm{CH} 30$ and 2N6-BA medium in 5 to 6 weeks under $25^{\circ} \mathrm{C}$ in dark conditions [56] Finally, the transgenic rice plants were regenerated in 2 to 10 weeks under $26^{\circ} \mathrm{C}$ light conditions in solidified MS medium [51,56,57]. All primers used in this study are listed in Table S1.

\subsection{Phenotypic Analysis}

To calculate the in vitro germination ratio, the viable pollen grains were germinated in solid and liquid pollen germination media. The liquid pollen germination medium consists of $20 \%$ sucrose, $10 \%$ polyethylene glycol 4000 (PEG4000), $3 \mathrm{mM} \mathrm{Ca}\left(\mathrm{NO}_{3}\right)_{2}, 40 \mathrm{mg} / \mathrm{L}$ $\mathrm{H}_{3} \mathrm{BO}_{3}$, and $10 \mathrm{mg} / \mathrm{L}$ thiamine (Vitamin B1) [33]. To make a solid germination medium, we added $1 \%$ agarose to the liquid medium. When the rice flowers reached anthesis, the fully mature pollen grains were collected in germination media immediately. Afterward, pollens on the germination media were incubated at $28^{\circ} \mathrm{C}$ for about $30 \mathrm{~min}$. We kept the humidity to prevent the germination medium from drying and observed the pollens using a BX61 microscope (Olympus, Tokyo, Japan). The pollen germination state and tube length were measured using Image J software [58]. More than 100 pollens were analyzed daily for a week. Ruthenium red and Calcofluor white were used for pectin, intine staining. All histochemical staining was incubated at room temperature for $15 \mathrm{~min}[31,33]$. The stained pollen grains were observed using the Olympus BX61 microscope.

\subsection{RNA Sequencing and Bioinformatic Analysis}

We sampled the anthers that contained pollen at tricellular stages. Pollens at different developmental stages were distinguished according to the size of the anther and the length of the filaments, referring to previous studies [59]. RNA-seq was performed on the Illumina platform in Macrogen (Korea). Raw data were rearranged using Cutadapt, mapped to the MSU7 reference genome, and then normalized by raw read counting to FeatureCounts and DESeq2 using the R package, following this reference [33,60,61]. GO enrichment features were selected by fold change threshold of $>2$, and the $p$-value $<0.05$. The GO plot is visualized using the R studio ggplot2 package. Upregulated genes and downregulated genes in the anther were inputted into the MapMan program. Regulation overview, metabolism overview, cellular response, and biotic and abiotic stress were selected for overview mapping. The bar graph was visualized using the R studio ggplot2 package. CAFRI-rice was used for expression analysis [62]. K-means clustering analysis was conducted by MultiExperiment Viewer software $[63,64]$

Supplementary Materials: The following are available online at https:/ /www.mdpi.com/article/10 $.3390 /$ ijms23010239/s1.

Author Contributions: K.-H.J. and Y.-J.K. designed the research project. E.-J.K., W.-J.H. and Y.-J.K. performed experiments. E.-J.K. analyzed data. E.-J.K. and K.-H.J. wrote the manuscript. All authors have read and agreed to the published version of the manuscript. 
Funding: This research was funded by the National Research Foundation of Korea (NRF) (NRF2021M3E5E6025387 to K.-H.J., 2019R1C1C1002636 to Y.-J.K., 2021R1A4A2001968 to Y.-J.K.); Rural Development Administration [PJ01492703 to K.-H.J.]. The APC was funded by the National Research Foundation of Korea (NRF) [NRF-2021M3E5E6025387 to K.-H.J.].

Institutional Review Board Statement: Not applicable.

Informed Consent Statement: Not applicable.

Data Availability Statement: All data are within the manuscript and its Supplementary files. Raw sequencing data and metadata have been deposited in ArrayExpress (https://www.ebi.ac.uk/ arrayexpress/experiments/E-MTAB-11246, accessed on 2 December 2021), with accession number E-MTAB-11246.

Acknowledgments: We thank Gynheung An and Jong-Seong Jeon at the Kyung Hee University for establishing the greenhouse and LMO field on the campus and providing valuable comments on this project.

Conflicts of Interest: The authors declare that the research was conducted without commercial or financial relationships that could be considered a potential conflict of interest.

\section{References}

1. Heslop-Harrison, J. Pollen Germination and Pollen-Tube Growth. Int. Rev. Cytol. 1987, 107, 1-78.

2. McCormick, S. Control of Male Gametophyte Development. Plant Cell. 2004, 16, S142-S153. [CrossRef] [PubMed]

3. Edlund, A.F.; Swanson, R.; Preuss, D. Pollen and Stigma Structure and Function: The Role of Diversity in Pollination. Plant Cell. 2004, 16, S84-S97. [CrossRef] [PubMed]

4. Koga, Y.; Akihama, T.; Fujimaki, H.; Yokoo, M. Studies on the Longevity of Pollen Grains of Rice, Oriza Sativa L I. Morphological Change of Pollen Grains After Shedding. Cytologia 1971, 36, 104-110. [CrossRef]

5. Firon, N.; Nepi, M.; Pacini, E. Water Status and Associated Processes Mark Critical Stages in Pollen Development and Functioning. Ann. Bot. 2012, 109, 1201-1214. [CrossRef]

6. Pacini, E.; Dolferus, R. Pollen Developmental Arrest: Maintaining Pollen Fertility in a World with a Changing Climate. Front. Plant. Sci. 2019, 10, 679. [CrossRef] [PubMed]

7. Franchi, G.G.; Bellani, L.; Nepi, M.; Pacini, E. Types of Carbohydrate Reserves in Pollen: Localization, Systematic Distribution and Ecophysiological Significance. Flora 1996, 191, 143-159. [CrossRef]

8. Datta, R.; Chamusco, K.C.; Chourey, P.S. Starch Biosynthesis during Pollen Maturation is Associated with Altered Patterns of Gene Expression in Maize. Plant. Physiol. 2002, 130, 1645-1656. [CrossRef] [PubMed]

9. Lee, S.; Kim, H.; Cho, J.; Nguyen, C.D.; Moon, S.; Park, J.E.; Park, H.R.; Huh, J.H.; Jung, K.; Guiderdoni, E. Deficiency of Rice Hexokinase HXK5 Impairs Synthesis and Utilization of Starch in Pollen Grains and Causes Male Sterility. J. Exp. Bot. 2020, 71, 116-125. [CrossRef]

10. Scofield, G.N.; Hirose, T.; Gaudron, J.A.; Furbank, R.T.; Upadhyaya, N.M.; Ohsugi, R. Antisense Suppression of the Rice Transporter Gene, OsSUT1, Leads to Impaired Grain Filling and Germination but does Not Affect Photosynthesis. Funct. Plant. Biol. 2002, 29, 815-826. [CrossRef]

11. Hirose, T.; Zhang, Z.; Miyao, A.; Hirochika, H.; Ohsugi, R.; Terao, T. Disruption of a Gene for Rice Sucrose Transporter, OsSUT1, Impairs Pollen Function but Pollen Maturation is Unaffected. J. Exp. Bot. 2010, 61, 3639-3646. [CrossRef]

12. Li, Y.; Faleri, C.; Geitmann, A.; Zhang, H.; Cresti, M. Immunogold Localization of Arabinogalactan Proteins, Unesterified and Esterified Pectins in Pollen Grains and Pollen Tubes of Nicotiana tabacum L. Protoplasma 1995, 189, 26-36. [CrossRef]

13. Denès, J.; Baron, A.; Renard, C.M.; Péan, C.; Drilleau, J. Different Action Patterns for Apple Pectin Methylesterase at pH 7.0 and 4.5. Carbohydr. Res. 2000, 327, 385-393. [CrossRef]

14. Micheli, F. Pectin Methylesterases: Cell Wall Enzymes with Important Roles in Plant Physiology. Trends Plant. Sci. 2001, 6, 414-419. [CrossRef]

15. Tian, G.; Chen, M.; Zaltsman, A.; Citovsky, V. Pollen-Specific Pectin Methylesterase Involved in Pollen Tube Growth. Dev. Biol. 2006, 294, 83-91. [CrossRef] [PubMed]

16. Kim, Y.; Jeong, H.Y.; Kang, S.; Silva, J.; Kim, E.; Park, S.K.; Jung, K.; Lee, C. Physiological Importance of Pectin Modifying Genes during Rice Pollen Development. Int. J. Mol. Sci. 2020, 21, 4840. [CrossRef] [PubMed]

17. Rounsley, S.D.; Ditta, G.S.; Yanofsky, M.F. Diverse Roles for MADS Box Genes in Arabidopsis Development. Plant. Cell. 1995, 7, $1259-1269$.

18. Riechmann, J.L.; Meyerowitz, E.M. MADS Domain Proteins in Plant Development. Biol. Chem. 1997, 378, 1079-1102.

19. Saedler, H.; Becker, A.; Winter, K.; Kirchner, C.; Theißen, G. MADS-Box Genes are Involved in Floral Development and Evolution. Acta Biochim. Pol. 2001, 48, 351-358. [CrossRef] [PubMed]

20. Zhang, J.; Cai, Y.; Yan, H.; Jin, J.; You, X.; Wang, L.; Kong, F.; Zheng, M.; Wang, G.; Jiang, L. A Critical Role of OsMADS1 in the Development of the Body of the Palea in Rice. J. Plant. Biol. 2018, 61, 11-24. [CrossRef] 
21. Honma, T.; Goto, K. Complexes of MADS-Box Proteins are Sufficient to Convert Leaves into Floral Organs. Nature 2001, 409, 525-529. [CrossRef]

22. Norman, C.; Runswick, M.; Pollock, R.; Treisman, R. Isolation and Properties of cDNA Clones Encoding SRF, a Transcription Factor that Binds to the C-Fos Serum Response Element. Cell 1988, 55, 989-1003. [CrossRef]

23. Kwantes, M.; Liebsch, D.; Verelst, W. How MIKC* MADS-Box Genes Originated and Evidence for their Conserved Function Throughout the Evolution of Vascular Plant Gametophytes. Mol. Biol. Evol. 2012, 29, 293-302. [CrossRef]

24. Kofuji, R.; Sumikawa, N.; Yamasaki, M.; Kondo, K.; Ueda, K.; Ito, M.; Hasebe, M. Evolution and Divergence of the MADS-Box Gene Family Based on Genome-Wide Expression Analyses. Mol. Biol. Evol. 2003, 20, 1963-1977. [CrossRef] [PubMed]

25. Honys, D.; Twell, D. Transcriptome Analysis of Haploid Male Gametophyte Development in Arabidopsis. Genome Biol. 2004, 5, 1-13. [CrossRef] [PubMed]

26. Verelst, W.; Twell, D.; de Folter, S.; Immink, R.; Saedler, H.; Münster, T. MADS-Complexes Regulate Transcriptome Dynamics during Pollen Maturation. Genome Biol. 2007, 8, R249. [CrossRef] [PubMed]

27. Theissen, G.; Saedler, H. Floral Quartets. Nature 2001, 409, 469-471. [CrossRef]

28. De Folter, S.; Angenent, G.C. Trans Meets Cis in MADS Science. Trends Plant. Sci. 2006, 11, 224-231. [CrossRef]

29. Verelst, W.; Saedler, H.; Munster, T. MIKC* MADS-Protein Complexes Bind Motifs Enriched in the Proximal Region of Late Pollen-Specific Arabidopsis Promoters. Plant. Physiol. 2007, 143, 447-460. [CrossRef]

30. Liu, Y.; Cui, S.; Wu, F.; Yan, S.; Lin, X.; Du, X.; Chong, K.; Schilling, S.; Theißen, G.; Meng, Z. Functional Conservation of MIKC*-Type MADS Box Genes in Arabidopsis and Rice Pollen Maturation. Plant. Cell. 2013, 25, 1288-1303. [CrossRef]

31. Kim, E.; Park, S.; Hong, W.; Silva, J.; Liang, W.; Zhang, D.; Jung, K.; Kim, Y. Genome-Wide Analysis of RopGEF Gene Family to Identify Genes Contributing to Pollen Tube Growth in Rice (Oryza Sativa). BMC Plant. Biol. 2020, 20, 95. [CrossRef] [PubMed]

32. Yao, W.; Li, G.; Yu, Y.; Ouyang, Y. funRiceGenes Dataset for Comprehensive Understanding and Application of Rice Functional Genes. Gigascience 2018, 7, gix119. [CrossRef]

33. Kim, Y.; Kim, M.; Hong, W.; Moon, S.; Kim, E.; Silva, J.; Lee, J.; Lee, S.; Kim, S.T.; Park, S.K. GORI, Encoding the WD40 Domain Protein, is Required for Pollen Tube Germination and Elongation in Rice. Plant J. 2021, 105, 1645-1664. [CrossRef]

34. Kim, Y.; Kim, M.; Hong, W.; Moon, S.; Tae Kim, S.; Ki Park, S.; Jung, K. OsMTD2-Mediated Reactive Oxygen Species (ROS) Balance is Essential for Intact Pollen Tube Elongation in Rice. Plant J. 2021, 107, 1131-1147. [CrossRef]

35. Chandran, A.K.N.; Hong, W.; Abhijith, B.; Lee, J.; Kim, Y.; Park, S.K.; Jung, K. Rice male gamete expression database (RMEDB): A web resource for functional genomic studies of rice male organ development. J. Plant. Biol. 2020, 63, 421-430. [CrossRef]

36. Niu, N.; Liang, W.; Yang, X.; Jin, W.; Wilson, Z.A.; Hu, J.; Zhang, D. EAT1 Promotes Tapetal Cell Death by Regulating Aspartic Proteases during Male Reproductive Development in Rice. Nat. Commun. 2013, 4, 1445. [CrossRef]

37. Liu, Z.; Lin, S.; Shi, J.; Yu, J.; Zhu, L.; Yang, X.; Zhang, D.; Liang, W. Rice no Pollen 1 (NP 1) is Required for Anther Cuticle Formation and Pollen Exine Patterning. Plant J. 2017, 91, 263-277. [CrossRef]

38. Ning, L.; Lin, Z.; Gu, J.; Gan, L.; Li, Y.; Wang, H.; Miao, L.; Zhang, L.; Wang, B.; Li, M. The Initial Deficiency of Protein Processing and Flavonoids Biosynthesis were the Main Mechanisms for the Male Sterility Induced by SX-1 in Brassica Napus. BMC Genom. 2018, 19, 806. [CrossRef] [PubMed]

39. Han, M.; Jung, K.; Yi, G.; Lee, D.; An, G. Rice Immature Pollen 1 (RIP1) is a Regulator of Late Pollen Development. Plant. Cell Physiol. 2006, 47, 1457-1472. [CrossRef]

40. Deng, F.; Li, Q.; Chen, H.; Zeng, Y.; Li, B.; Zhong, X.; Wang, L.; Ren, W. Relationship between Chalkiness and the Structural and Thermal Properties of Rice Starch After Shading during Grain-Filling Stage. Carbohydr. Polym. 2021, 252, 117212. [CrossRef]

41. Zhang, K.; Song, Q.; Wei, Q.; Wang, C.; Zhang, L.; Xu, W.; Su, Z. Down-regulation of Os SPX 1 Caused Semi-male Sterility, Resulting in Reduction of Grain Yield in Rice. Plant. Biotechnol. J. 2016, 14, 1661-1672. [CrossRef]

42. Yim, S.; Khare, D.; Kang, J.; Hwang, J.; Liang, W.; Martinoia, E.; Zhang, D.; Kang, B.; Lee, Y. Postmeiotic Development of Pollen Surface Layers Requires Two Arabidopsis ABCG-Type Transporters. Plant. Cell Rep. 2016, 35, 1863-1873. [CrossRef]

43. Goetz, M.; Godt, D.E.; Guivarc'h, A.; Kahmann, U.; Chriqui, D.; Roitsch, T. Induction of Male Sterility in Plants by Metabolic Engineering of the Carbohydrate Supply. Proc. Natl. Acad. Sci. USA 2001, 98, 6522-6527. [CrossRef] [PubMed]

44. Waller, L.N.; Fox, N.; Fox, K.F.; Fox, A.; Price, R.L. Ruthenium Red Staining for Ultrastructural Visualization of a Glycoprotein Layer Surrounding the Spore of Bacillus Anthracis and Bacillus Subtilis. J. Microbiol. Methods 2004, 58, 23-30. [CrossRef]

45. Herth, W.; Schnepf, E. The Fluorochrome, Calcofluor White, Binds Oriented to Structural Polysaccharide Fibrils. Protoplasma 1980, 105, 129-133. [CrossRef]

46. Moon, S.; Jung, K. First Steps in the Successful Fertilization of Rice and Arabidopsis: Pollen Longevity, Adhesion and Hydration. Plants 2020, 9, 956. [CrossRef] [PubMed]

47. Leroux, C.; Bouton, S.; Kiefer-Meyer, M.; Fabrice, T.N.; Mareck, A.; Guénin, S.; Fournet, F.; Ringli, C.; Pelloux, J.; Driouich, A. PECTIN METHYLESTERASE48 is Involved in Arabidopsis Pollen Grain Germination. Plant. Physiol. 2015, 167, 367-380. [CrossRef]

48. Hepler, P.K.; Kunkel, J.G.; Rounds, C.M.; Winship, L.J. Calcium Entry into Pollen Tubes. Trends Plant. Sci. 2012, 17, 32-38. [CrossRef] [PubMed]

49. Iwano, M.; Shiba, H.; Miwa, T.; Che, F.; Takayama, S.; Nagai, T.; Miyawaki, A.; Isogai, A. Ca2 Dynamics in a Pollen Grain and Papilla Cell during Pollination of Arabidopsis. Plant. Physiol. 2004, 136, 3562-3571. [CrossRef] 
50. Liu, L.; Zheng, C.; Kuang, B.; Wei, L.; Yan, L.; Wang, T. Receptor-Like Kinase RUPO Interacts with Potassium Transporters to Regulate Pollen Tube Growth and Integrity in Rice. PLoS Genet. 2016, 12, e1006085. [CrossRef]

51. Moon, S.; Chandran, A.K.N.; Kim, Y.; Gho, Y.; Hong, W.; An, G.; Lee, C.; Jung, K. Rice RHC Encoding a Putative Cellulase is Essential for Normal Root Hair Elongation. J. Plant. Biol. 2019, 62, 82-91. [CrossRef]

52. Aboul-Maaty, N.A.; Oraby, H.A. Extraction of High-Quality Genomic DNA from Different Plant Orders Applying a Modified CTAB-Based Method. Bull. Natl. Res. Cent. 2019, 43, 25. [CrossRef]

53. Kim, E.; Kim, Y.; Hong, W.; Lee, C.; Jeon, J.; Jung, K. Genome-Wide Analysis of Root Hair Preferred RBOH Genes Suggests that Three RBOH Genes are Associated with Auxin-Mediated Root Hair Development in Rice. J. Plant. Biol. 2019, 62, $229-238$. [CrossRef]

54. Schmittgen, T.D.; Livak, K.J. Analyzing Real-Time PCR Data by the Comparative C T Method. Nat. Protoc. $2008,3,1101$. [CrossRef] [PubMed]

55. Xie, K.; Minkenberg, B.; Yang, Y. Boosting CRISPR/Cas9 Multiplex Editing Capability with the Endogenous tRNA-Processing System. Proc. Natl. Acad. Sci. USA 2015, 112, 3570-3575. [CrossRef] [PubMed]

56. Lee, S.; Jeon, J.; Jung, K.; An, G. Binary Vectors for Efficient Transformation of Rice. J. Plant. Biol. 1999, 42, 310-316. [CrossRef]

57. Jeon, J.; Lee, S.; Jung, K.; Jun, S.; Jeong, D.; Lee, J.; Kim, C.; Jang, S.; Lee, S.; Yang, K. T-DNA Insertional Mutagenesis for Functional Genomics in Rice. Plant J. 2000, 22, 561-570. [CrossRef]

58. Schneider, C.A.; Rasband, W.S.; Eliceiri, K.W. NIH Image to ImageJ: 25 Years of Image Analysis. Nat. Methods. 2012, 9, 671-675. [CrossRef]

59. Moon, S.; Oo, M.M.; Kim, B.; Koh, H.; Oh, S.A.; Yi, G.; An, G.; Park, S.K.; Jung, K. Genome-Wide Analyses of Late Pollen-Preferred Genes Conserved in various Rice Cultivars and Functional Identification of a Gene Involved in the Key Processes of Late Pollen Development. Rice 2018, 11, 28. [CrossRef]

60. Liao, Y.; Smyth, G.K.; Shi, W. FeatureCounts: An Efficient General Purpose Program for Assigning Sequence Reads to Genomic Features. Bioinformatics 2014, 30, 923-930. [CrossRef]

61. Love, M.I.; Huber, W.; Anders, S. Moderated Estimation of Fold Change and Dispersion for RNA-Seq Data with DESeq2. Genome Biol. 2014, 15, 550. [CrossRef] [PubMed]

62. Hong, W.; Kim, Y.; Kim, E.; Kumar Nalini Chandran, A.; Moon, S.; Gho, Y.; Yoou, M.; Kim, S.T.; Jung, K. CAFRI-Rice: CRISPR Applicable Functional Redundancy Inspector to Accelerate Functional Genomics in Rice. Plant J. 2020, 104, 532-545. [CrossRef] [PubMed]

63. Howe, E.; Holton, K.; Nair, S.; Schlauch, D.; Sinha, R.; Quackenbush, J. Mev: Multiexperiment viewer. In Biomedical Informatics for Cancer Research; Springer: Boston, MA, USA, 2010; pp. 267-277.

64. Liu, T.; Xu, X.; Leng, W.; Xue, Y.; Dong, J.; Jin, Q. Analysis of Gene Expression Changes in Trichophyton Rubrum after Skin Interaction. J. Med. Microbiol. 2014, 63, 642-648. [CrossRef] [PubMed] 